\title{
Study of Liver Metabolism in Glucose-6- Phosphatase Deficiency (Glycogen Storage Disease Type 1A) by P-31 Magnetic Resonance Spectroscopy
}

\author{
R. D. OBERHAENSLI, B. RAJAGOPALAN, D. J. TAYLOR, G. K. RADDA, J. E. COLLINS, AND \\ J. V. LEONARD \\ MRC Facility for Clinical Magnetic Resonance, John Radcliffe Hospital, Oxford and Institute of Child Health, \\ London, England
}

\begin{abstract}
Liver metabolism of two patients (aged 15 and 23 yr) was studied by $P$-31 magnetic resonance spectroscopy at 1.9 tesla. The P-31 spectra of liver showed the resonances of phosphomonoesters (including sugar phosphates), inorganic phosphate $\left(\mathbf{P}_{\mathrm{i}}\right)$, phosphodiesters (e.g. glycerophosphorylcholine, glycerophosporylethanolamine), and ATP. These resonances were quantified by expressing their peak areas in $\mathrm{mM}$ (assuming that ATP concentrations in normal liver is $2.5 \mathrm{mM}$ ) or as a ratio relative to the area of the phosphodiester resonance. After an overnight fast liver phosphomonoesters in patients were 2.6 and 1.6 $\mathrm{AU}$, respectively (controls $1.1 \pm 0.5$, mean \pm $2 \mathrm{SD}, n=17)$. At the same time liver $P_{i}$ was decreased in patients to 1.3 and 1.0 , respectively (controls $1.8 \pm 0.8$ ). Based on chemical shift measurements the increase in phosphomonoesters could be attributed to accumulation of sugar phosphates (mainly glycolytic intermediates). After $1 \mathrm{~g} / \mathrm{kg}$ oral glucose, hepatic sugar phosphates decreased in patients by 64 and $40 \%$, respectively, and reached normal levels (on the absolute intensity scale); whereas liver $\mathbf{P}_{i}$ increased by 130 and $40 \%$, respectively. Liver $P_{i}$ levels remained elevated in both patients 30 min after ingestion of glucose. Liver sugar phosphates and $P_{i}$ did not change in control subjects $(n=4)$ after glucose. In contrast to some previous reports, we have found accumulation of glycolytic intermediates in the liver of glucose-6-phosphatase-deficient patients during fasting. In these patients high levels may enhance the activity of residual glucose-6-phosphatase thus increasing hepatic glucose production and reducing the degree of hypoglycemia during fasting. Hyperuricemia is another serious complication of glucose-6phosphatase deficiency and was present in both patients. Levels of $P_{i}$ are known to regulate synthesis and breakdown purines. The changes in liver $P_{i}$ during fasting and refeeding in these patients may stimulate production of uric acid and contribute to the hyperuricemia. (Pediatr Res 23: 375380,1988 )
\end{abstract}

\section{Abbreviations}

P-31, phosphorus 31

$P_{i}$, inorganic phosphate

Glucose-6-P, glucose-6-phosphate

Received June 15, 1987; accepted December 9, 1987

Correspondence and reprint requests Prof. G. K. Radda, Department of Biochemistry, South Parks Road, Oxford OX1 3QU, England.

Supported by the Medical Research Council and the Department of Health and Social Security. R.D.O. was supported by the Swiss National Science Foundation. J.E.C. was an Action Research Training Fellow.
Glucose-6-phosphatase is necessary for the release of free glucose from glucose-6-P derived from glycogenolysis or gluconeogenesis. The link between glucose-6-phosphatase deficiency and its metabolic manifestations is not clear (1). Fasting hypoglycemia, a typical feature of glucose-6-phosphatase deficiency, can be accounted for by the block in glucose release. However, the striking ability of glucose-6-phosphatase-deficient patients to maintain from $30-100 \%$ of the normal glucose output during fasting has been difficult to explain in view of the reduction in hepatic glucose-6-phosphatase activity by more than $95 \%(2,3)$ (Collins JE, Leonard JV, unpublished observations). A second clinical problem in glucose-6-phosphatase-deficient patients is hyperuricemia and gout (4-9). It has been suggested that the block in hepatic glucose release leads to accumulation of glucose6-P during fasting. Glucose-6-P would overflow into the pentose phosphate shunt, increasing the formation of ribose-5-P and ultimately uric acid (4). It has also been speculated that trapping of $P_{i}$ in glucose-6-P could reduce the cytosolic concentration of $P_{i}$. At low $P_{i}$ adenosine monophosphate deaminase, a key enzyme of purine degradation, would be activated and uric acid production would increase (6-9). However, accumulation of hepatic glucose-6-P during fasting has never been convincingly demonstrated in glucose-6-phosphatase deficiency. Elevated glucose-6$P$ levels in liver biopsy samples of patients with glucose-6phosphatase deficiency have been reported in only two studies $(10,11)$ and these results could not be confirmed by independent investigators who reanalyzed part of the same liver samples (12). Direct experimental evidence for depletion of liver $P_{i}$ in glucose6-phosphatase deficiency during fasting has never been provided.

P-31 magnetic resonance spectroscopy provides a new approach to the investigation of hepatic metabolism of $\mathrm{P}_{\mathrm{i}}$ and sugar phosphates. We have shown previously that the levels of sugar phosphates, $\mathrm{P}_{\mathrm{i}}$, and ATP, can be assessed by P-31 magnetic resonance spectroscopy in healthy and diseased liver (13-15). Herein we used this technique to investigate the hepatic metabolism of two patients with glucose-6-phosphatase deficiency.

\section{MATERIALS AND METHODS}

Study protocol. Patient 1 was a 15 -yr-old boy who presented at the age of 5 months with hypoglycemia and hepatomegaly. The glycogen content of a liver biopsy was increased and glucose6-phosphatase activity was not detectable (Prof. A. D. Patrick, Institute of Child Health, London, England). At the time of study his growth was normal and he was treated with cornstarch by day and continuous nasogastric feeds at night. Patient 2 was a 23 -yr-old man who presented at the age of $3 \mathrm{yr}$ with failure to thrive. Liver biopsy showed an increase in liver glycogen with 
undetectable glucose-6-P activity. At the time of the study he had been on a normal diet for $4 \mathrm{yr}$, having been on nocturnal nasogastric feeds for the previous 4 yr. Both patients were receiving allopurinol. Subjects were studied after a fast, patient 1 after $6 \mathrm{~h}$ and patient 2 overnight. After a baseline P-31 spectrum of the liver was obtained, the subject was taken out of the magnet and a blood sample was drawn. The subject was repositioned in the magnet and further control spectra were obtained for $10 \mathrm{~min}$. The subjects drank $1 \mathrm{~g} / \mathrm{kg}$ body weight glucose dissolved in 200 $\mathrm{ml}$ water and P-31 spectra were recorded for another $30 \mathrm{~min}$. At the end of the experiment a second blood sample was collected. The patients' baseline spectra were compared with control data obtained in 17 healthy volunteers aged $24-43$ yr. Four volunteers aged 24-36 yr served as controls for the oral glucose tolerance test. The study protocol was approved by the local ethics committee and informed consent was obtained from patients and control subjects before the experiment.

Magnetic resonance spectroscopy was done as described previously $(13,14)$. P-31 spectra were obtained at 1.9 tesla using a surface coil $(8 \mathrm{~cm}$ in diameter) for data acquisition. Magnetic field profiling was used to localize signals from the liver. P-31 spectra with acceptable signal to noise (signal to noise ratio $>$ 10:1) were obtained in 4-10 min. The resonances were identified from their chemical shifts using the signal from water as the frequency reference. $\mathrm{pH}$ was calculated from the chemical shift of $\mathrm{P}_{\mathrm{i}}$ as described previously (14). Because liver does not contain phosphocreatine (the common reference compound in $\mathrm{pH}$ determinations), the signal from water was used as the internal frequency reference. Spectra were collected and plotted under identical conditions in each subject and were quantified in two ways. First, the areas of individual peaks were measured and expressed as a ratio compared to area of the diester peak. Second, the areas of the $\beta$-phosphate peaks of ATP were measured in the controls. The arithmetic mean of these areas was set equal to. 2.5 AU. [ATP concentration in human liver is about $2.5 \mu \mathrm{mol} / \mathrm{g}$ wet weight $(7,16)$.] This calibration factor was then used to express the peak areas of all metabolites in this study in the same AU. Neither absolute signal intensities nor metabolite ratios were corrected for saturation effects due to incomplete spin-lattice relaxation. At the pulse conditions used $\left(180^{\circ}\right.$ pulse at coil center and interpulse delay of $1 \mathrm{~s}$ ) only ATP was fully relaxed whereas phosphomonoesters, $\mathrm{P}_{\mathrm{i}}$, and phosphodiesters were about 70, 80, and $50 \%$, respectively, of the unsaturated signal intensity. Inasmuch as complete spin-lattice relaxation measurements were too time consuming to obtain in these experiments (17) possible changes in relaxation behavior due to the metabolic abnormality were assessed by acquiring data at two different interpulse delays $(0.1$ and $1.0 \mathrm{~s})$. The effect of the spin-lattice relaxation time on the peak intersites was estimated by calculating the ratio signal intensity at $0.1 \mathrm{~s}$ interpulse delay/signal intensity at $1.0 \mathrm{~s}$ interpulse delay.

Blood samples for the determination of xanthine and hypoxanthine were deproteinized with perchloric acid (final concentration $4 \%$ ) and immediately frozen. Xanthine and hypoxanthine were measured by high-performance liquid chromatography (18). Because the changes in blood levels of hypoxanthine closely reflected those in xanthine, only the hypoxanthine data will be presented herein. Blood glucose, uric acid, and $P_{i}$ were determined by multichannel analyzer in the hospital laboratory.

Data presentation. Because only two patients were investigated, statistical analysis of the data was not performed. The normal range was defined as mean $\pm 2 \mathrm{SD}$.

\section{RESULTS}

Figure 1 shows liver P-31 spectra of a control subject and patient 1 after fasting. Signal intensities for phosphodiesters and ATP in liver of patients were within the normal range when expressed on the absolute scale shown in Table 1. Determination of the spin-lattice relaxation factors in patient 2 excluded signif-

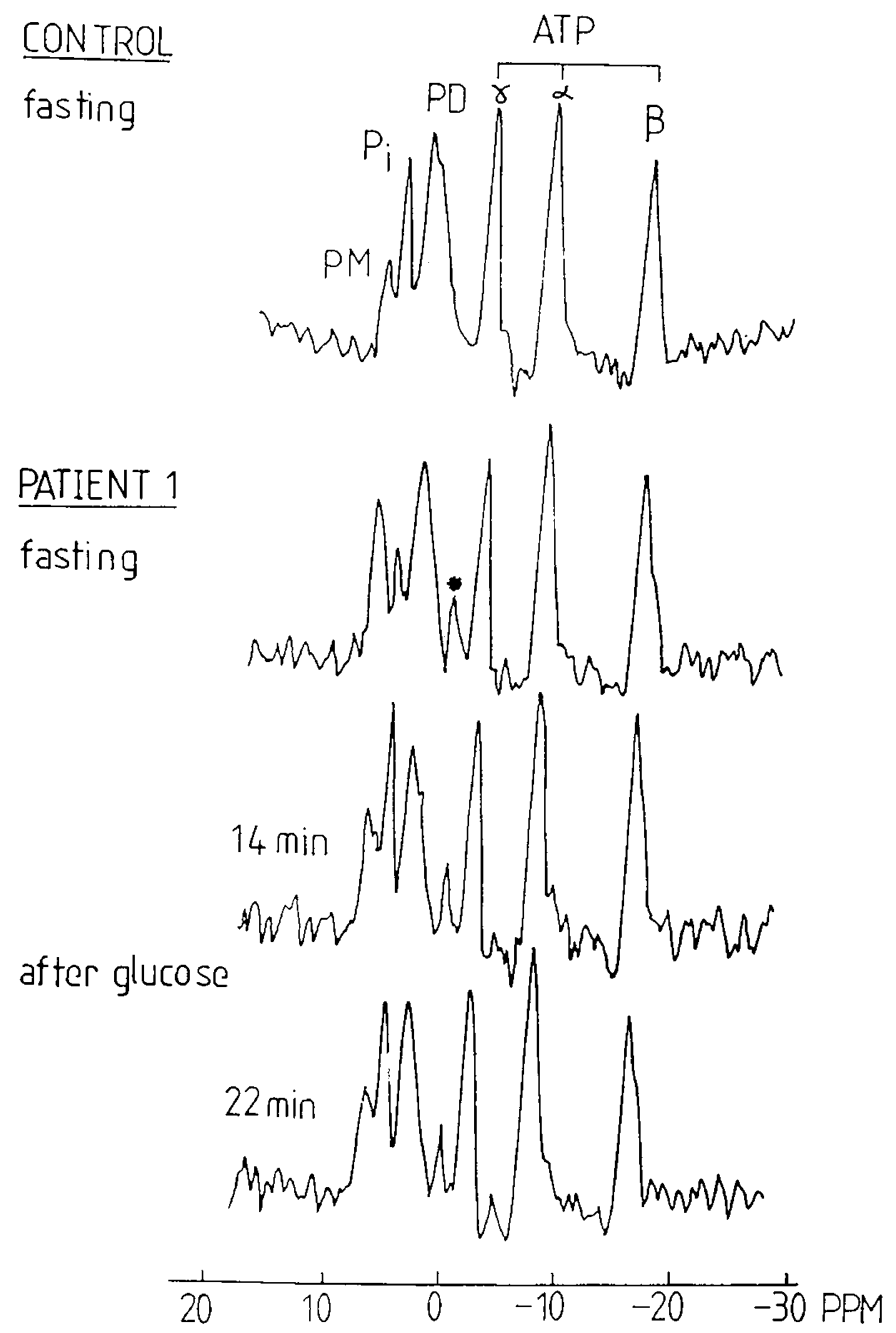

Fig. 1. Liver P-31 spectra of a control subject (512 accumulations and a glucose-6-phosphatase-deficient patient (360 accumulations). $P M$, phosphomonoesters; $P D$, phosphodiesters. * phosphocreatine due to small contamination of the spectrum with signals from skeletal muscle (intercostals or diaphragm).

icant changes in the spin-lattice relaxation time of the measured metabolites. These factors could not be determined in patient 1 for technical reasons. Intracellular liver $\mathrm{pH}$ was normal in both patients. After fasting overnight the phosphomonoesters were increased in patient 1 when measured on both the absolute scale and also relative to the phosphodiester. The $P_{i}$ peak was low normal on the arbitrary scale but reduced when expressed relative to the phosphodiester peak. Patient 2 had broadly similar changes. The phosphomonoesters were increased relative to diesters but not on the absolute scale. By contrast $\mathrm{P}_{\mathrm{i}}$ was low normal on both scales. In both patients the $\mathrm{P}_{\mathrm{i}}$ /phosphomonoester ratio is markedly disturbed (Table 1).

After the glucose load the liver P-31 spectra of controls did not change significantly whereas marked effects were observed in the liver spectra of both patients (Figs. 1 and 2). The phosphomonoesters gradually decreased in both patients after oral glucose, and at $30 \mathrm{~min}$ the intensity of the peak was 1.1 in patient 1 and 0.9 in patient 2 . This was within the normal range $(1.3 \pm$ 0.5 , mean $\pm 2 \mathrm{SD}, n=4$ ). The decrease in the phosphomonoester resonance was evident when the data were expressed relative to the signal intensity before glucose ingestion. After glucose an upfield shift of the phosphomonoester peak by about $0.5 \mathrm{ppm}$ was measured in the liver spectra of both patients.

Liver $P_{i}$ increased in both patients after oral glucose. Liver $P_{i}$ of patient 1 reached its highest concentration $14 \mathrm{~min}$ after 
Table 1. Liver metabolites after fasting overnight

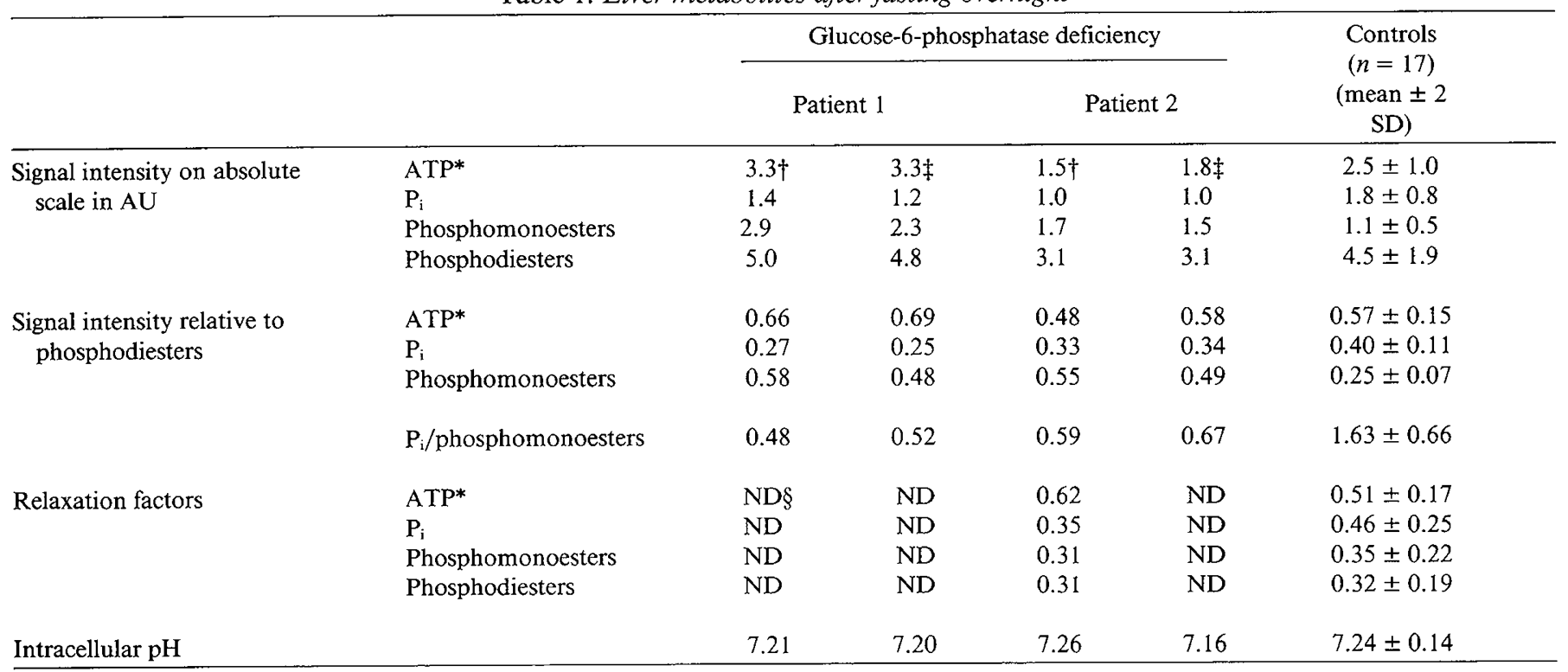

* Derived from $\beta$-phosphate of ATP.

$\dagger+$ Data were obtained in two independent experiments separated by $15-20 \mathrm{~min}$ ( $\dagger$ first run, $\ddagger$ second run). $\S$ Not determined.

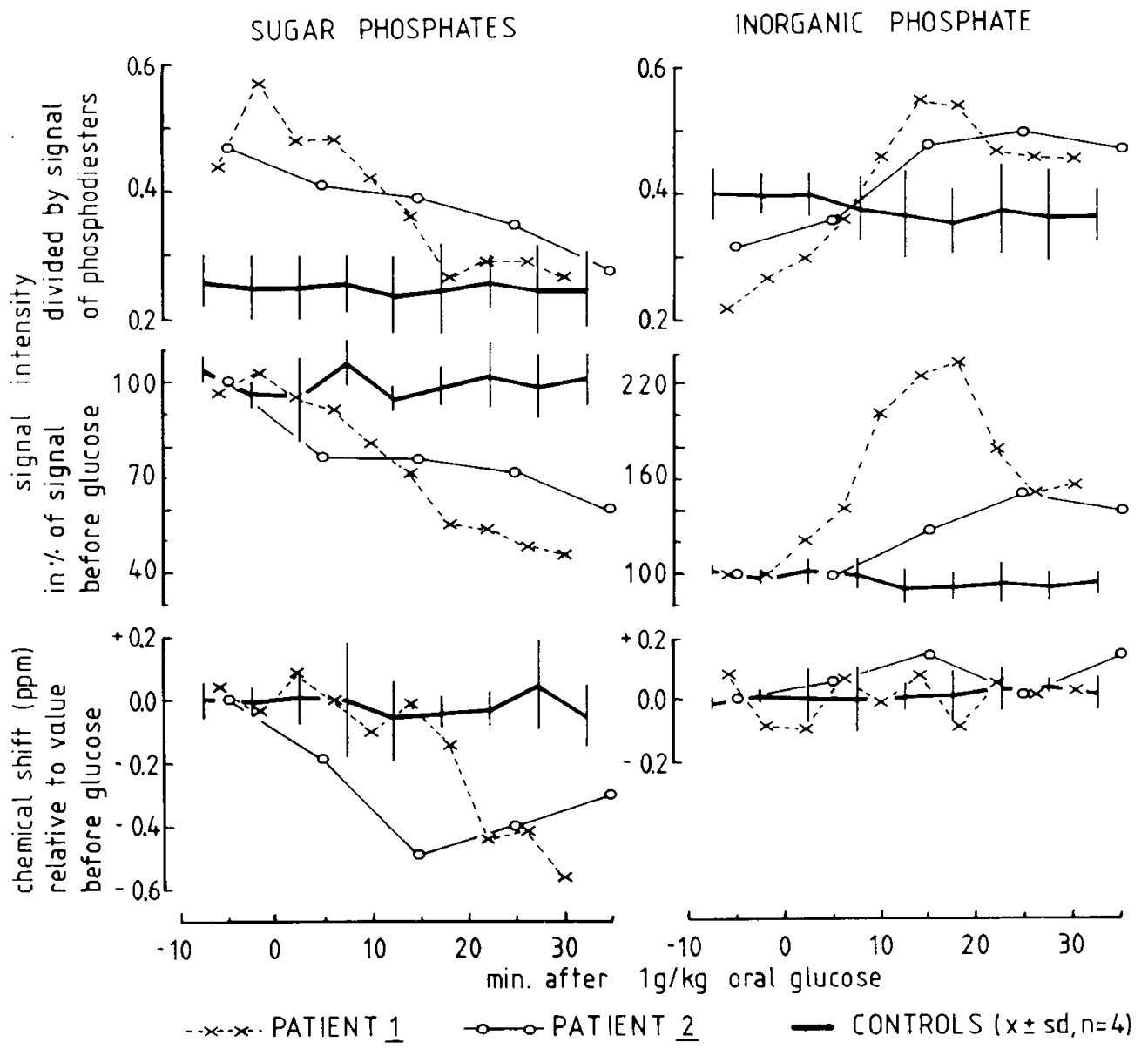

Fig. 2. Effect of oral glucose on liver $P_{i}$ and sugar phosphate in glucose-6-phosphatase-deficient patients.

glucose, and $\mathrm{P}_{\mathrm{i}}$ was clearly increased when expressed relative to phosphodiesters (patient $1,0.55$, controls, $0.36 \pm 0.10$ ) or on the absolute intensity scale (patient 1, 2.8, controls, $2.0 \pm 0.6$ ). Liver $\mathrm{P}_{\mathrm{i}}$ after ingestion of glucose did not reach as high a value on the absolute intensity scale in patient 2 as in patient 1 . However, when the data were expressed relative to phosphodiesters, liver $P_{\mathrm{i}}$ in patient 2 was also clearly elevated 30 min after glucose (patient 2, 0.48, controls, $0.37 \pm 0.08$ ). The glucose-induced rise in liver $\mathrm{P}_{\mathrm{i}}$ was best seen when the data were expressed as percentage of the signal intensity before glucose ingestion. In con- 
trast to phosphomonoesters the chemical shift of $P_{i}$ stayed constant after glucose excluding significant changes in liver $\mathrm{pH}$. The intensity of the ATP and phosphodiester resonances stayed constant throughout the experiment (data not shown).

The patients' blood glucose concentration was low before the glucose load (patient 1,2.9 mM, patient 2, $3.5 \mathrm{mM}$, controls 4.3 $\pm 0.3 \mathrm{mM}$, mean $\pm 2 \mathrm{SD}$ ). A total of $45 \mathrm{~min}$ after glucose it rose to $9.3 \mathrm{mM}$ in patient 1 and $9.2 \mathrm{mM}$ in patient 2 (controls, 5.4 $\pm 2.6 \mathrm{mM}$ ). The elevated blood glucose we observed in both patients $45 \mathrm{~min}$ after the glucose load presumably reflects the impaired glucose tolerance reported to occur in glucose-6-phosphatase deficiency (4). Plasma $P_{i}$ was normal in patients after fasting overnight (patient $1,1.3 \mathrm{mM}$, patient $2,1.4 \mathrm{mM}$, controls, $1.1 \pm 0.2 \mathrm{mM})$. It did not change significantly after ingestion of glucose (patient 1, 1.7 mM, patient 2, $1.2 \mathrm{mM}$, controls, $1.0 \pm$ $0.2 \mathrm{mM}$ ). While fasting both patients showed increased blood levels of hypoxanthine (patient $1,8.7 \mu \mathrm{M}$, patient $2,11.9 \mu \mathrm{M}$, controls $2.5 \pm 0.6$ ). Hypoxanthine decreased $45 \mathrm{~min}$ after oral glucose to $5.2 \mu \mathrm{M}$ in patient 1 and $7.4 \mu \mathrm{M}$ in patient 2 (whereas insignificant change was observed in controls). Plasma uric acid levels increased in both patients (patient $1,699 \mu \mathrm{M}$, patient 2 , $737 \mu \mathrm{M}$, normal range, $200-450 \mu \mathrm{M}$ ) but did not decrease after glucose. Plasma uric acid levels were not expected to be a reliable index of purine breakdown herein because both patients were treated, albeit inadequately, with a xanthine oxidase inhibitor.

\section{DISCUSSION}

Interpretation of P-31 spectra. The phosphomonoester peak contains the resonances of phosphorylated carbohydrates including hexose, pentose, and triose phosphates (19). It also contains the resonances of phosphocholine and phosphoethanolamine (19). The phosphodiester resonance consists of the signals of glycerophosphorylcholine and glycerophosphorylethanolamine (19). Mobile phospholipids in membranes are also thought to contribute substantially to this resonance (20). Under the experimental conditions of our study the $P_{i}$ peak corresponds to intracellular (mainly cytosolic) $\mathrm{P}_{\mathrm{i}}$ in liver cells (13). Due to the small difference in chemical shift of about $0.5 \mathrm{ppm}$ the purine and pyrimidine nucleotides cannot be separated by P-31 magnetic resonance spectroscopy at 1.9 tesla. Inasmuch as ATP is the most abundant of these high energy phosphates the peaks of purine and pyrimidine nucleotides are herein referred to as ATP peaks.

Assessment of absolute concentrations by magnetic resonance spectroscopy in vivo is difficult. Herein we quantified the data in three ways. First, the phosphodiester resonance was used as internal reference. Second, we measured the absolute signal intensity of the P-31 resonances of the various metabolites. This intensity depends on many parameters: the volume of the sample and its position relative to the main coil from which the signal is derived, the instrumental parameters, and the relaxation behavior of the P-31 nuclei. In our standard experimental protocols the variations among subjects was $20-30 \%$ and on repeat measurements of a given subject it was $20 \%$. Because the factor needed to convert signal intensity into absolute concentrations was not known, the metabolite concentration had to be expressed in AU. However, as patient and control data were collected under the same conditions they can be compared directly. Third, in serial studies signal intensity was expressed as a percentage of the baseline value.

The results suggest that there is a decrease in $P_{i}$ and increase in phosphomonoesters in the livers of the glucose-6-phosphatasedeficient patients after fasting. Inasmuch as we only studied two patients, these changes are difficult to demonstrate as statistically significant in the direct measurement of absolute intensities. The conclusions, however, are supported by the observation that after glucose phosphomonoester decreases and $P_{i}$ increases in the patients but not in controls. The changes measured do not result from changes in the spin-lattice relaxation times.
We can estimate the change in concentrations in $P_{i}$ and phosphomonoesters if we assume that the concentrations of ATP in the liver of patients was the same as determined by chemical methods in normal liver $(2.5 \mu \mathrm{mol} / \mathrm{g}$ wet weight $)$. We also have to correct for the saturation of the magnetic resonance signals. The increase in phosphomonoesters in patients after fasting corresponded to $0.9-1.8 \mu \mathrm{mol} / \mathrm{g}$ wet weight. After the glucose load the decrease in liver $P_{i}$ was about $0.6-2.0 \mu \mathrm{mol} / \mathrm{g}$ wet weight. Although this change in $P_{i}$ appears small in relation to the chemically measured $P_{i}$ in liver [5 $\mu \mathrm{mol} / \mathrm{g}$ wet weight (16)], it is important to remember that $\mathrm{P}-31$ magnetic resonance spectroscopy only detects $P_{i}$ dissolved in cell water and not $P_{i}$ bound to cellular structures (21).

Analysis of the chemical shift data suggests that the large phosphomonoester peak in the liver spectra of patients was due to accumulation of sugar phosphates. The phosphomonoester peak is composed of the resonances of sugar phosphates and phosphocholine and phosphoethanolamine. The resonances of these two groups of compounds are separated by $0.6 \mathrm{ppm}$ and could not be resolved in our studies (19). However, the chemical shift of the phosphomonoester peak is dependent on the relative contribution of these two resonances. For example, the decrease in the sugar phosphate concentration in the liver of both patients leads to an upfield shift consistent with the contribution of the phosphocholine phosphoethanolamine becoming more prominent (Fig. 2). This shift is not a result of a decrease in liver $\mathrm{pH}$ because liver $\mathrm{pH}$ as determined from the chemical shift of $\mathrm{P}_{\mathrm{i}}$ did not change.

It is important to determine whether the accumulation of hepatic sugar phosphates was due to glycolytic intermediates or to the ribose-5-phosphate of adenosine monophosphate or inosine monophosphate, reflecting increased ATP synthesis or breakdown. Hexose-6-phosphate and pentose-5-phosphate cannot be distinguished by $\mathrm{P}-31$ magnetic resonance spectroscopy at 2 tesla because the differences in chemical shift are too small. If the decrease in sugar phosphates after a glucose load resulted from that in ribose-5-phosphate of nucleoside monophosphates we would expect either an increase in ATP in the liver spectra or a surge of xanthine, hypoxanthine, and uric acid in the blood. Neither was observed and it is therefore likely that the accumulated sugar phosphate in the liver of patients was mainly due to glycolytic intermediates.

Herein we demonstrate three abnormalities in liver metabolism in glucose-6-phosphatase deficiency: accumulation of phosphomonoesters (probably glycolytic intermediates), depletion of intracellular $\mathrm{P}_{\mathrm{i}}$ during fasting, and elevation of $\mathrm{P}_{\mathrm{i}}$ after refeeding. Accumulation of glycolytic intermediates in liver in glucose-6phosphatase deficiency during fasting has been the subject of controversy. Concentrations of liver glucose-6-P have been reported to range from normal to 10 times normal $(10-12)$. These conflicting results probably reflect the inadequacies of methods for the measurement of liver metabolites in biopsy specimens rather than true biologic variability (12). It has been speculated that depletion of liver $P_{i}$ could occur in glucose-6-phosphatasedeficient patients during fasting as a result of $P_{i}$ trapping in sugar phosphates, but there has been no experimental evidence for this (6-8).

We estimate that sugar phosphates increased in the livers of both patients by about $0.9-1.8 \mu \mathrm{mol} / \mathrm{g}$ wet weight during fasting. This corresponds to $2-4 \mathrm{mM}$ assuming the cytosolic water space to be $0.5 \mathrm{ml} / \mathrm{g}$ wet weight (22). Measurement on biopsies from the livers of patients with glucose-6-phosphatase deficiency shows that the concentrations of glucose-6-P, fructose-6-phosphate, and fructose-1,6-phosphate increased to a total of $2.2 \mu \mathrm{mol} / \mathrm{g}$ wet weight after stimulation by glucagon. Glucose-6-P contributed $65 \%$ to this increase in sugar phosphates (7). It is therefore likely that the increase we observed by magnetic resonance spectroscopy was partly due to glucose-6-P.

The high glucose-6-P concentration in the liver during fasting may explain why patients with glucose-6-phosphatase deficiency 
can maintain $50 \%$ or more of the normal glucose output during fasting despite the enzyme deficiency. Normal glucose-6-phosphatase activity has been reported to be between 3-10 $\mu \mathrm{mol} /$ $\mathrm{min} / \mathrm{g}$ liver, (23) indicating that a normal adult liver of $1500 \mathrm{~g}$ could release $4.5-15.0 \mathrm{mmol} /$ glucose $/ \mathrm{min}$. This is six to 20 times that necessary to maintain euglycemia in normal adults after an overnight fast (24). Glucose-6-phosphatase has a high $\mathrm{Km}$ for its substrate in rat liver (about $2 \mathrm{mM}$ ) (25). This together with the lack of experimental evidence of a hormonal control mechanism has led to the concept that the metabolic flux through glucose6-phosphatase is mainly controlled by the concentration of glucose-6-P which varies typically in rat liver from $0.05-0.15 \mu \mathrm{mol} /$ $\mathrm{g}$ wet weight between the fed and fasted state $(26,27)$.

The residual enzyme activity of the liver of patients with glucose-6-phosphatase deficiency is undetectable or very low [the mean in one large series was $0.17 \mu \mathrm{mol} / \mathrm{min} / \mathrm{g}$ liver (28)]. Even at this activity the increase in glucose-6-P in the patients during a fast may lead to measurable glucose production. It is also possible that the glucose-6-phosphatase activity measured in vitro is less than that in vivo because the enzyme is labile (29) and membrane bound.

It has also been suggested that glucose can be released by a glucose-6-P/glycogen (futile) cycle $(1,11,30)$ involving breakdown of glycogen by amylo-1,6,glucosidase (debrancher enzyme). Because glycogen structure is normal in glucose-6-phosphatase deficiency, about 10-12 molecules of glucose would have to be cycled to release 1 molecule of glucose (31). To account for glucose production of $0.2 \mu \mathrm{mol} / \mathrm{g}$ liver $/ \mathrm{min}$ in glucose-6phosphatase-deficient patients during fasting (3) glucose cycling would have to proceed at a rate of $2.5 \mu \mathrm{mol} / \mathrm{g}$ liver $/ \mathrm{min}$ implying near maximal rates of glycogen synthesis and breakdown (32). However, the accumulation of glycolytic intermediates we observed in both patients excludes active glycogen synthesis during fasting because during rapid glycogen synthesis the concentration of glucose-6-P is decreased $(26,27)$. We conclude that on the present evidence a futile cycle between glucose-6-P and glycogen is unlikely to account for the total glucose output.

After the glucose load the concentration of hepatic sugar phosphates decreased rapidly to normal levels in the patients indicating that the accumulated glycolytic intermediates were metabolized and the metabolic block at the level of glucose-6phosphatase had become less significant. Isotope dilution studies have demonstrated that hepatic glucose production is completely suppressed at high intestinal glucose absorption rates in glucose6-phosphatase-deficient patients as well as in controls (32) (thus the metabolic flux through glucose-6-phosphatase is low after glucose). The disappearance of hepatic sugar phosphates after the glucose load was presumably due to the activation of glycogen synthase $(26,27,32)$ which leads to rapid consumption of glucose-6-P $(26,27)$.

The low $P_{i}$ concentration in liver of both patients during fasting was unexpected. Whereas, clearly $P_{i}$ is converted to sugar phosphate, on the basis of animal experiments we would have expected that intracellular $P_{i}$ is rapidly replenished from the extracellular $P_{i}$ pool $(33,34)$. This either means that in patients the $P_{i}$ influx is slow or that the high extracellular/intracellular gradient is actively maintained. This gradient can be estimated if we assume that the plasma $P_{i}$ concentration is a measure of the extracellular $P_{i}$ concentration and that $P-31$ signal intensities for $P_{i}$ on the absolute scale relate to intracellular $P_{i}$ concentrations. Inasmuch as the extracellular $P_{i}$ concentrations in both patients remained normal during fasting, as previously reported $(5,6)$, the extracellular/intracellular $P_{i}$ gradient was about twice as high in patients than in controls (see Table 1 for intracellular $\mathrm{P}_{\mathrm{i}}$ ).

The clinical course of glucose-6-phosphatase deficiency is complicated by hyperuricemia and gout (4-9). Detailed studies on uric acid turnover indicate that increased production and decreased renal clearance of uric acid contribute to the hyperuricemia $(5,6,9)$. The intracellular $P_{i}$ level is closely related to uric acid production in liver by regulating purine nucleotide synthesis and degradation. The control step in purine nucleotide breakdown is catalyzed by adenosine monophosphate deaminase (3537) and in purine synthesis by phosphoribosylpyrophosphate synthase (38). Both enzymes are inhibited by $\mathrm{P}_{\mathrm{i}}$ at physiologic concentrations in vitro (36-38).

In the two patients the liver $\mathrm{P}_{\mathrm{i}}$ was depleted during fasting and it increased by 40 and $180 \%$, respectively, after glucose. The low liver $P_{i}$ concentration during fasting could lead to disinhibition of adenosine monophosphate deaminase and $5^{\prime}$-nucleotidase and promote purine degradation to uric acid. The high liver $\mathbf{P}_{\mathrm{i}}$ levels after refeeding may increase phosphoribosylpyrophosphate synthase activity and enhance purine synthesis. It is conceivable that liver $P_{i}$ in glucose-6-phosphatase-deficient patients undulates between high and low levels in relation to feeding. These fluctuations in liver $P_{i}$ would provide a stimulus for purine synthesis or breakdown and eventually lead to hyperuricemia. Accumulation of glucose-6-P could contribute further to the increased production of uric acid by enhancing the formation of ribose-5-phosphate from glucose-6-P in the pentose phosphate shunt (39). The increased supply of ribose-5-phosphate to phosphoribosylpyrophosphate synthase could trigger purine synthesis (38).

In conclusion, we have found by P-31 magnetic resonance spectroscopy that glycolytic intermediates accumulate in the liver of patients with glucose-6-phosphatase deficiency during fasting. High levels of glucose-6-P may enable these patients to overcome in part the deleterious effects of the block in hepatic glucose release and achieve glucose production rates that meet the metabolic demands of glucose-dependent organs (e.g. brain) during fasting $(2,3)$. The finding of large fluctuations in intracellular $P_{i}$ in the liver may be directly related to the increase in uric acid production typically seen in these patients (4).

Acknowledgments. The authors thank Dr. E. Harmsen for the determination of blood xanthine and hypoxanthine and A. Thomas and L. McFarlane for technical assistance.

\section{REFERENCES}

1. Williams JC 1986 Nutritional goals in glycogen storage disease. N Engl J Med 314:709-710

2. Tsalikian E, Simmons P, Gerich JE, Howard C, Haymond MW 1984 Glucose production and utilization in children with glycogen storage disease type $\mathrm{I}$. Am J Physiol 247:E513-E519

3. Havel RJ, Balasse EO, Williams HE, Kane JP, Segel N 1969 Splanchnic metabolism in von Gierke's disease. Trans Assoc Am Phys LXXXII:305323

4. Howel RR, Ashton DM, Wyngarden JB 1962 Glucose-6-phosphatase deficiency glycogen storage disease. Studies on the interrelationships of carbohydrate, lipid and purine abnormalities. Pediatrics 29:553-565

5. Kelly NW, Rosenbloom FM, Seegmiller JE, Howell RR 1968 Excessive production of uric acid in type I glycogen storage disease. J Paediatr 72:488498

6. Roe TF, Kogut MD 1977 The pathogenesis of hyperuricemia in glycogen storage disease, type I. Pediatr Res 11:664-669

7. Greene HL, Wilson FA, Hefferan P, Terry AB, Moran JB, Slonim AE, Claus TH 1978 ATP depletion, a possible role in the pathogenesis of hyperuricemia in glycogen storage disease type $\mathrm{I}$. J Clin Invest 62:321-328

8. Cohen JL, Vinik A, Faller J, Fox IH 1985 Hyperuricemia in glycogen storage disease type I. J Clin Invest 75:251-257

9. Fine RN, Strauss J, Donnell GN 1966 Hyperuricemia in glycogen storage disease type I. Am J Dis 112:572-576

10. Oeckerman PA 1965 Assay by spectrofluorimetric method of glucose-6-phosphate in glycogen storage disease type I. Clin Chim Acta 12:445-452

11. Brante G, Kajiser K, Oeckerman PA 1964 Glycogenosis type I in four siblings. Acta Pediatr Scand [Suppl] 157:1-24

12. Hers HG 1964 Glycogen storage disease. In: Levine R, Luft R (eds) Advances in Metabolic Disease, vol I. Academic Press, New York, pp 1-45

13. Oberhaensli RD, Galloway GJ, Taylor DJ, Bore PJ, Radda GK 1986 Assessment of human liver metabolism by phosphorus-31 magnetic resonance spectroscopy. Br J Radiol 59:695-699

14. Oberhaensli RD, Galloway GJ, Hilton-Jones D, Bore PJ, Styles P, Taylor DJ, Rajagopalan B, Radda GK 1987 The study of human organs by phosphorus31 topical magnetic resonance spectroscopy. Br J Radiol 60:367-373

15. Oberhaensli RD, Hilton-Jones D, Bore PJ, Hands LJ, Rampling RP, Radda GK 1986. Biochemical investigation of human tumours in vivo with phosphorus-31 magnetic resonance spectroscopy. Lancet 2:8-10

16. Bode JC, Zelder O, Rumpelt HJ, Wittkamp U 1973 Depletion of liver adenosine phosphates and metabolic effects of intravenous infusion of fructose 
and sorbitol in man and the rat. Eur J Clin Invest 3:436-441

17. Blackledge MJ, Oberhaensli RD, Styles P, Radda GK 1987 Measurements of in vivo relaxation rates and spectral editing in human organs using rotating frame depth selection. J Magn Reson 71:331-336

18. Harmsen E, de Jong JW, Serruys PW 1981 Hypoxanthine production by ischemic heart demonstrated by high pressure liquid chromatography of blood purine nucleosides and oxypurines. Clin Chim Acta 115:73-84

19. Cohen SM 1983 Simultaneous C-13 and P-31 NMR studies of perfused rat liver. J Biol Chem 258:14294-14308

20. De Kruijff B, van den Besselaar AM, Cullis PR, van den Bosch $H$, Deenen LLM 1978 Evidence for isotropic motion of phospholipids in liver microsomal membranes. A P-31 NMR study. Biochim Biophys Acta 514:1-8

21. Radda GK, Taylor DJ 1985 Applications of nuclear magnetic resonance spectroscopy in pathology. Int Rev Exp Pathol 27:1-55

22. Akerboom TB, Bookelman H, Zuurendonk PF, Meer R, Tager JM 1978 Intramitochondrial and extramitochondrial concentrations of adenine nucleotides and inorganic phosphate in isolated hepatocytes from fasted rats. Eur J Biochem 84:413-420

23. McCabe ERB, Melvin TR, O'Brien D, Montgomery RR, Robinson WA, Bhasker C, Brown BI. 1980 Neutropenia in a patient with type 1 B glycogen storage disease: in vitro response to lithium chloride. J Pediatr 97:944-946

24. Rizza R, Haymond H, Cryer P, Gerich J 1979 Differential effects of epinephrine on glucose production and disposal in man. Am $\mathbf{J}$ Physiol 6:E356-E362

25. Nordlie RC, Arion WJ 1964 Evidence for the common identity of glucose-6phosphatase, inorganic pyrophosphatase and pyrophosphate-glucose transferase. J Biol Chem 239:1680 -1686

26. Hers HG, Hue $L 1983$ Gluconeogenesis and related aspects of glycolysis. Annu Rev Biochem 52:617-653

27. Newgard CB, McGarry JD 1984 Evidence for suppression of glucose-6-phosphatase with carbohydrate feeding. Diabetes 33:192-195

28. Brown BI 1985 Diagnosis of glycogen storage. In: Wapnir RA (ed) Congenital
Metabolic Diseases. Diagnosis and Treatment. Marcel Dekker Inc., New York, pp 227-250

29. Spencer-Peet J, Norman ME, Lake BD, McNamara J, Patrick AD 1971 Hepatic glycogen storage disease. Clinical and laboratory findings in 23 cases. $Q \mathrm{~J}$ Med 40:95-114

30. Senior B, Loredan L 1968 Study of liver glycogenoses with particular reference to the metabolism of intravenously administered glycerol. $N$ Engl $J$ Med 279:958-965

31. Illingworth B, Cori GT 1952 Structure of glycogens and amylopectins. Normal and abnormal human glycogens. J Biol Chem 199:653-660

32. Bishop JS, Larner J 1967 Rapid activation-inactivation of liver uridine diphosphate glucose-glycogen transferase and phosphorylase by insulin and glucagon in vivo. Proc Natl Acad Sci USA 242:1354-1356

33. Schwenk WF, Haymond MW 1986 Optimal rate of enteral glucose administration in children with glycogen storage disease type I. N Engl J Med 314:682-685

34. Sestoft L, Kristensen LO 1979 Determination of unidirectional fluxes of phosphate across plasma membrane in isolated perfused rat liver. Am $\mathrm{J}$ Physiol 5:C202-C210

35. Till U, Bros D, Frunder H 1969 Orthophosphate turnover in the extracellular and intracellular space of mouse liver. Eur J Biochem 11:541-548

36. van den Berghe $G$, Bontemps F, Hers HG 1980 Purine catabolism in rat hepatocytes. Biochem J 188:913-920

37. van den Berghe G, Bronfman M, Vaneste R, Hers HG 1977 The mechanism of ATP depletion in the liver after a load of fructose. A kinetic study of liver adenylate deaminase. Biochem $\mathbf{J}$ 162:601-609

38. Chapman AG, Atkinson DF 1973 Stabilization of adenylate energy charge by the adenylate deaminase reaction. J Biol Chem 248:8309-8312

39. Fox IH, Kelly WN 1971 Human phosphoribosylpyrophosphate synthase. J Biol Chem 246:5739-5748 